\title{
MATRICES IN LINEAR MECHANICAL SYSTEMS
}

\author{
Marvin Marcus (1) \\ (received June 25, 1962)
}

In a recent interesting pape $\mathrm{r}$ [1] on the stability of Iinear mechanical systems S. P. Diliberto discusses certain reduction theorems for symmetric and skew-symmetric Hamiltonian matrices with respect to symplectic orthogonal similarity. In what follows it is shown that a unified simple a rgument will handle both of the se problems together and then as an example it is indicated how the argument can be also used to obtain the reduction theorem for symplectic orthogonal matrices.

If $\mathrm{A}$ and $\mathrm{B}$ are matrices $\mathrm{A} \otimes \mathrm{B}$ will denote their Kronecker product. Let $\mathrm{E}$ be the 2-square matrix $\left(\begin{array}{rr}0 & 1 \\ -1 & 0\end{array}\right)$ and $I_{n}$ be the $n$-square identity matrix. Define $J=E \otimes I_{n}$ so that $J^{2}=E^{2} \otimes I_{n}=-I_{2} \otimes I_{n}=-I_{2 n}$. A real $2 n$-squa re matrix $A$ is called Hamiltonian if $(J A)^{\prime}=J A ; A$ is called symplectic if $\mathrm{A}^{\prime} \mathrm{JA}=\mathrm{J}$. If the following matrices a re all real $\mathrm{n}$-square then a block multiplication ea sily establishes

LEMMA 1.

(1) $\left(\begin{array}{cc}R & M \\ L & -R^{\prime}\end{array}\right)\left(\begin{array}{cc}U & V \\ -V & U\end{array}\right)=\left(\begin{array}{cc}X & Y \\ Z & -W\end{array}\right)$

where

$$
\begin{aligned}
& (R+i M)(U+i V)=X+i Y, X=R U-M V, Y=M U+R V \\
& \left(R^{\prime}+i L\right)(U+i V)=W+i Z, W=R^{\prime} U-L V, Z=L U+R^{\prime} V
\end{aligned}
$$

(1) This research was supported by the U.S. Air Force Office of Scientific Research.

Canad. Math. Bull. vol. 5, no. 3, September 1962. 
Note that $\mathrm{A}$ is Hamiltonian if and only if

$$
A=\left(\begin{array}{cc}
R & M \\
L & -R^{\prime}
\end{array}\right) \text {, }
$$

in which $L$ and $M$ are symmetric; for, if

(4) $\quad A=\left(\begin{array}{ll}A_{11} & A_{12} \\ A_{21} & A_{22}\end{array}\right)$ then

$A^{\prime} J^{\prime}=J A$ implies that $A_{21}^{\prime}=A_{21}, A_{12}^{\prime}=A_{12}, A_{11}^{\prime}=-A_{22}$

and so $A$ has the form (3).

LEMMA 2. A is symplectic orthogonal if and only if

$$
A=I_{2} \otimes U+E \otimes V
$$

in which $U+i V$ is n-square unitary.

Proof. Since $A^{\prime}=A^{-1}$ it follows that $A$ must commute with $\mathrm{J}$. Using the notation in (4) it follows immediately by a block multiplication that $A_{11}=A_{22}, A_{12}=-A_{21}$ and $A$ has the form (5). Now

$$
\begin{aligned}
& A A^{\prime}=\left(I_{2} \otimes U+E \otimes V\right)\left(I_{2} \otimes U^{\prime}+E^{\prime} \otimes V^{\prime}\right) \\
& =I_{2} \otimes\left(U U^{\prime}+V V^{\prime}\right)+E \otimes\left(V U^{\prime}-U V^{\prime}\right) .
\end{aligned}
$$

If $A A^{\prime}=I_{2 n}=I_{2} \otimes I_{n}$ then $U U^{\prime}+V V^{\prime}=I_{n}$ and $V U^{\prime}-U V^{\prime}=0$. These a re precisely the necessary and sufficient conditions that $U+i V$ be unitary as can be checked by multiplying out $(U+i V)(U+i V) *=I_{n}$.

In the following theorem $\mathrm{T}$ is an orthogonal symplectic matrix and $A$ is Hamiltonian. Diliberto's two reduction theorems are contained in

\section{THEOREM 1. If $A$ is:}

(a) skew-symmetric then the re exists a real diagonal matrix $D$ such that 
(6) $T^{-1} A T=E \otimes D ;$

(b) symmetric then there exists a real diagonal matrix D such that

(7) $\mathrm{T}^{-1} \mathrm{AT}=\mathrm{F} \otimes \mathrm{D}$

where $F=\left(\begin{array}{rr}1 & 0 \\ 0 & -1\end{array}\right)$.

Proof. (a): The matrix $A$ has the form (3) and if in addition $A^{\prime}=-A$ it follows that $R^{\prime}=-R$ and $L=-M$ is symmetric. Thus $A=I_{2} \otimes R+E \otimes M$. Let $H=R+i M$ and notice that $H^{*}=R^{\prime}-i M^{\prime}=-R-i M=-H$. Thus $H$ is skew-hermitian and by the diagonalization theorem for skewhermitian matrices there is a unitary $U+i V, U, V$ real, and a real diagonal $D$ such that $(R+i M)(U+i V)=(U+i V)(i D)$ $=-V D+i U D$. By (2) $X=-V D, Y=U D$. AIso $\left(R^{\prime}+i L\right)(U+i V)$ $=(-R-i M)(U+i V)=-(R+i M)(U+i V)=V D-i U D$ so $W=V D$ and $\mathrm{Z}=-\mathrm{UD}$. Let $\mathrm{T}=\mathrm{I}_{2} \otimes \mathrm{U}+\mathrm{E} \otimes \mathrm{V}$ and by Lemma 2, $T$ is orthogonal symplectic. Then from (1) $A T=\left(\begin{array}{rr}-V D & U D \\ -U D & -V D\end{array} \mid=T(E \otimes D)\right.$, completing the proof of $(a)$.

(b): If $\mathrm{A}$ is symmetric then from (3) again it follows immediately that $R^{\prime}=R$ and $M=L$ is also symmetric. Then

$$
A=F \otimes R+E \otimes L .
$$

Let $\mathrm{H}$ be the complex symmetric matrix $\mathrm{R}+\mathrm{iL}$. A classical result of I. Schur [2] states that a unitary matrix $S=U+i V$ exists such that

$$
S^{\prime}(R+i L) S=D
$$

in which $\mathrm{D}$ is a real diagonal matrix (whose main diagonal elements are the singular values of $R+i L)$. Now $\left(S^{\prime}\right)^{-1}=\bar{S}=U-i V$ so that $(R+i M)(U+i V)=(U-i V) D=U D-i V D$. From (2) again $X=U D, \quad Y=-V D$. Also $\left(R^{\prime}+i L\right)(U+i V)$ $=(R+i M)(U+i V)=U D-i V D$ so $W=U D, \quad Z=-V D$. 
Once again $\mathrm{T}=\mathrm{I}_{2} \otimes \mathrm{U}+\mathrm{E} \otimes \mathrm{V}$ is orthogonal symplectic and from (1),

$$
A T=\left(\begin{array}{rr}
U D & -V D \\
-V D & -U D
\end{array}\right)=T(F \otimes D)
$$

completing the proof of (b).

Another result that follows by the same kind of argument is

THEOREM 2. If $A$ is symplectic and orthogonal then there exists a symplectic orthogonal $T$ such that

$$
\mathrm{T}^{-1} \mathrm{AT}=\mathrm{I}_{2} \otimes \mathrm{D}+\mathrm{E} \otimes \mathrm{K}
$$

in which $\mathrm{D}=\operatorname{diag}\left(\mathrm{d}_{1}, \ldots, \mathrm{d}_{\mathrm{n}}\right)$ is a real diagonal matrix and $K=\operatorname{diag}\left(-\sqrt{1-d_{1}^{2}}, \ldots \pm \pm \sqrt{1-d_{n}^{2}}\right)$.

Proof. If $A$ is symplectic and orthogonal then by Lemma $2 \mathrm{~A}=\mathrm{I}_{2} \otimes \mathrm{R}+\mathrm{E} \otimes \mathrm{L}$ in which $\mathrm{R}+\mathrm{iL}$ is unitary. By the diagonalization theorem for unitary matrices obtain a unitary matrix $U+i V$ for which $(R U-L V)+i(L U+R V)$ $=(R+i L)(U+i V)=(U+i V)(D+i K)=(U D-V K)+i(V D+U K)$, in which $\mathrm{D}$ and $\mathrm{K}$ are diagonal matrices. Let $\mathrm{T}$ be the orthogonal symplectic matrix $I_{2} \otimes U+E \otimes V$ and compute that

$$
\begin{aligned}
A T & =\left(I_{2} \otimes R+E \otimes L\right)\left(I_{2} \otimes U+E \otimes V\right) \\
& =I_{2} \otimes(R U-L V)+E \otimes(L U+R V) \\
& =I_{2} \otimes(U D-V K)+E \otimes(V D+U K) \\
& =\left(I_{2} \otimes U+E \otimes V\right)\left(I_{2} \otimes D+E \otimes K\right) \\
& =T\left(I_{2} \otimes D+E \otimes K\right) .
\end{aligned}
$$

The fact that $A$ is orthogonal implies that $D^{2}+K^{2}=I_{n}$ and the proof is complete. 


\section{REFERENCES}

1. S. P. Diliberto, On stability of Iinear mechanical systems, Office of Naval Research Technical Report, Prepared under contract NONR 222(88). University of California, Berkeley. May, (1962).

2. I. Schur, Ein Satz ueber quadratische Formen mit komplexen Koeffizienten, Amer. J. Math. 67, (1945), 472.

University of California, Santa Barbara and

University of British Columbia 